\title{
Studies of (GaAl)As injection lasers operating with an optical fiber resonator
}

\author{
Luis Figueroa, Kam Y. Lau, ") Huan W. Yen, and Amnon Yariv b) \\ Hughes Research Laboratories, Malibu, California 90265
}

(Received 13 December 1979; accepted for publication 29 February 1980)

\begin{abstract}
The characteristics of an optical fiber external resonator in conjunction with (GaAl)As stripe geometry lasers are described. We have observed a 6-10\% reduction in the threshold current and have obtained $150 \mathrm{ps}$ pulses at gigahertz repetition rates. The fiber resonator has also been used to quench self-pulsations in a (GaAl)As injection laser. In order to explain many of our results we have used a model that uses the conventional semiconductor rate equations modified by the addition of saturable electron traps and the effects of the external cavity. Our results predict many of the self-locking effects observed in injection lasers operating in an external cavity.

Furthermore, the degree of self-locking will be a strong function of the external cavity length and the density of saturable absorbers.
\end{abstract}

PACS numbers: 42.55.Px, 42.80.Lt

\section{INTRODUCTION}

In recent years the generation of short optical pulses at $\mathrm{GHz}$ rates has become a subject of great practical interest. The high-speed pulses can be used to study fast optical processes, ${ }^{1}$ as optical clocks in optical logic circuits, ${ }^{2}$ and for the phase locking of microwave semiconductor sources. ${ }^{3,4}$ Conventional gas, ${ }^{5}$ solid state, ${ }^{6}$ and dye lasers ${ }^{7}$ have achieved picosecond pulses at moderately high repetition rates, but they remain large and cumbersome, a major drawback in applications where light weight is desirable. Recently, the mode locking of a (GaAl)As injection laser using an external optical cavity has been reported. ${ }^{8-10}$ Pulses approximately $20 \mathrm{ps}$ in width at $3-\mathrm{GHz}$ repetition rates were observed. Other experiments have shown that it is possible to generate short pulses (less than $180 \mathrm{ps)}$ ) at $\mathrm{GHz}$ repetition rates by using a self-pulsing injection laser. ${ }^{11}$ The frequency of the pulsation could be partially controlled by using an external cavity. Earlier experiments have shown that $100 \%$ sinusoidal modulation can be obtained by operating the laser in an external cavity. ${ }^{12}$ Recently, an external cavity was used to suppress self-pulsations in injection lasers. ${ }^{11,13,14}$ We believe the study of injection lasers operating in an external cavity is an important subject which can lead to a clarification of the mechanisms responsible for pulsations. In this paper we describe our study of injection lasers operating in an external cavity. In particular we discuss a novel external resonator using a multimode graded index fiber. The laser-fiber combination can be relatively compact, rugged, light weight, and mechanically stable, and therefore potentially practical. Furthermore, it can be used to generate picosecond pulses at $\mathrm{GHz}$ rates and to suppress self-pulsations in injection lasers. In Sec. II we discuss the light output characteristics of the laser-fiber combination. An effective reflectivity is defined for the laser-fiber combination and the reduction of the threshold current is calculated and compared to experiment. In Sec. III we discuss our theory on self-locking effects in injection lasers operating in an external cavity. We use the electron trap model, in the manner of Copeland, and include the effects of an external reflector. We find that many of the seemingly different earlier experimental results are explained within the scope of our present model. In Sec. IV we give experimental results and compare them to the calculated results of Sec. III. Good qualitative agreement is obtained. In Sec. $V$ we summarize our results and provide some additional discussions.

\section{EXPERIMENTAL CONFIGURATION AND THE LIGHT OUTPUT CHARACTERISTICS OF AN EXTERNAL FIBER RESONATOR}

In Fig. 1 we show the experimental arrangement for the external cavity geometry. The devices used are stripe geometry GaAs double-heterostructure lasers furnished by RCA Labs. These lasers are symmetric ridge-waveguide lasers similar to those reported by Botez. ${ }^{15} 16$ The lasers operate in the fundamental lateral and transverse mode for the range of currents used in the experiment $\left(I<1.1 I_{\text {th }}\right)$. We operate the lasers $\mathrm{cw}$ at all times. The external resonator consists of a piece of multimode graded index optical fiber with numerical aperture equal to 0.14 . Both ends are cleaved and one end of the fiber is coated with $2500 \AA$ of Au to form a reflector. A spherical lens (shown in Fig. 2) is formed on the other end of the fiber using the thermal melting technique. ${ }^{17}$ A typical

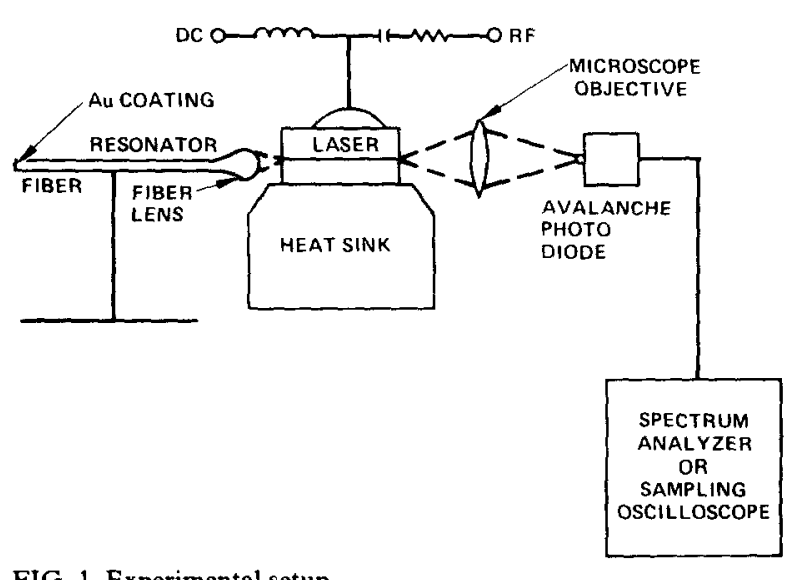

FIG. 1. Experimental setup.
"California Institute of Technology, Pasadena, Calif. 90265.

h'California Institute of Technology, Pasadena, Calif. 90265.
TIG. 1. Experimental setup. 


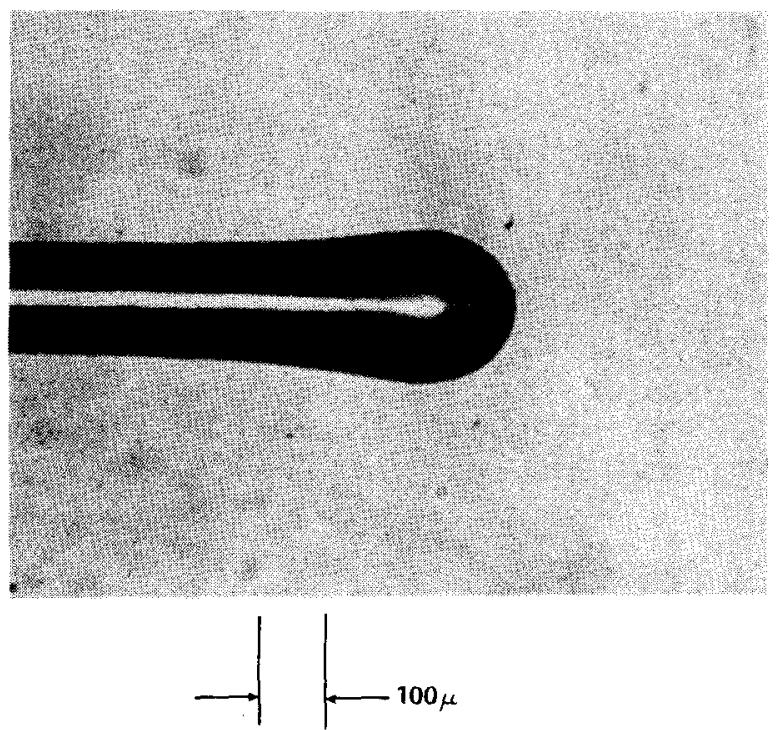

FIG. 2. Top view of optical fiber showing a lens tip.

radius of curvature for the lens is $80-120 \mu$. The experimental arrangement consists of mounting the laser at the end of a microstrip bias tee with a $47-\Omega$ resistor placed in series with the rf input to provide impedance matching. The fiber is placed inside a capillary tube which is mounted on an $x-y-z$ stage with provision for rotation about one of the axes. The alignment is performed using PZT controlled micrometers. The light output from the second facet of the laser is collected and focused onto a Si Avalanche Diode (ED55) using a $10 \times$ microscope objective. The rise time of the Avalanche Diode used is around 150-200 ps. The if input to the laser is provided by an HP sweep oscillator (8690B). The maximum rf power going into the laser is estimated to be a few $\mathrm{mW}$.

The output from the Avalanche Diode is fed to either an HP spectrum analyzer (8565A) or a Tektronix sampling oscilloscope (Samp. Head 7511).

In Fig. 3 we show a typical light output versus current characteristic for an external fiber resonator (EFR). For

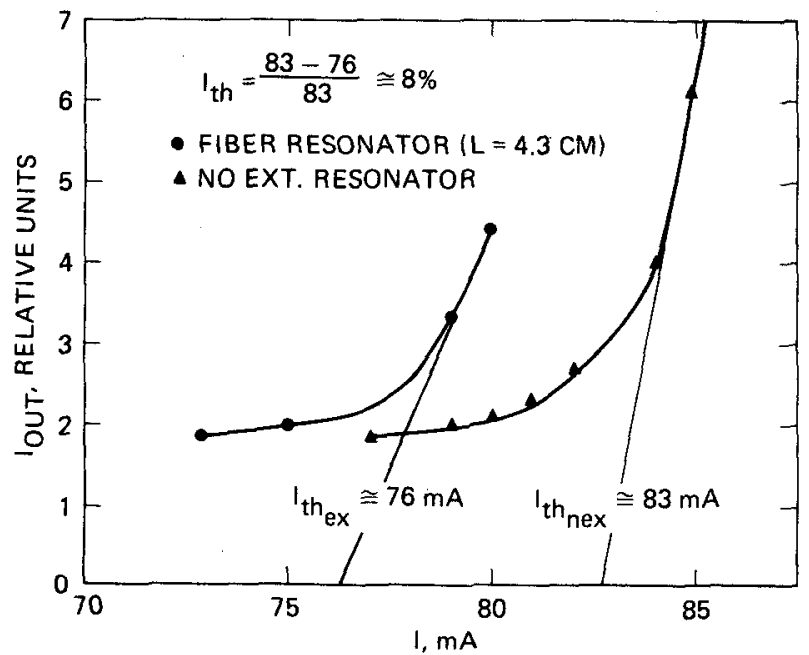

FIG. 3. Light output vs current for the optical fiber external resonator configuration. comparison we also show the light-output characteristics with no external resonator (ER) present. An important figure of merit for an ER is the threshold reduction factor $K{ }^{18}$ which is given by

$$
K=J_{\text {th ER }} / J_{\text {th }} \text {, }
$$

where $J_{\text {th ER }}$ and $J_{\text {th }}$ are the threshold current densities with and without the ER, respectively. In order to see how the value of $K$ depends on the laser and external resonator parameters we can write down an expression relating the laser gain $g$ to the current density $J$. First, we assume a liner relationship ${ }^{19,20}$ between gain and current density given by

$$
g=\left[\beta\left(\eta_{1} J\right) / d\right]-\alpha_{0},
$$

where $\beta$ is the gain constant, $\eta_{I}$ is the internal efficiency, $d$ is the active region thickness, and $\alpha_{0}$ is a loss constant. The mode gain $G$, which includes the effect of transverse optical confinement $\Gamma$, is

$$
G=\Gamma g \text {. }
$$

At threshold we have

$$
J_{\mathrm{th}}=\left(\beta \eta_{t}\right)^{-1}\left[\alpha_{0} d+(d / \Gamma) G_{\mathrm{th}}\right] .
$$

The threshold mode gain is given by the familiar expression

$$
G_{\mathrm{th}}=\alpha_{\mathrm{fc}}+(1 / 2 l) \ln \left(1 / R R_{\mathrm{eff}}\right),
$$

where $\alpha_{\mathrm{fc}}$ is the distributed loss, $l$ is the diode length, $R$ is the facet reflectivity, and $R_{\text {eff }}$ is defined as the total fraction of light reflection back into the laser from the external and diode mirror combination. We should note that by using an $R_{\text {eff }}$ we are assuming a uniform photon density in the external cavity. This assumption is adequate for $\mathrm{cw}$ operation, but it breaks down in the description of short optical pulses. Using Eqs. (4) and (5) we find that $K$ can be expressed as

$$
K=\frac{\alpha_{0} d+(d / \Gamma)\left[\alpha_{\mathrm{fc}}+(1 / 2 l) \ln (1 / R)\left(1 / R_{\mathrm{eff}}\right)\right]}{\alpha_{0} d+(d / \Gamma)\left[\alpha_{\mathrm{fc}}+(1 / l) \ln (1 / R)\right]} .
$$

The effective reflectivity can be expressed as ${ }^{21}$

$$
R_{\text {eff }}=\frac{R+2 R^{1 / 2} R_{f}^{1 / 2} \cos 2 \delta+R_{f}}{1+2 R^{1 / 2} R_{f}^{1 / 2} \cos 2 \delta+R_{f} R},
$$

where $2 \delta$ is the round trip phase delay of wave propagation

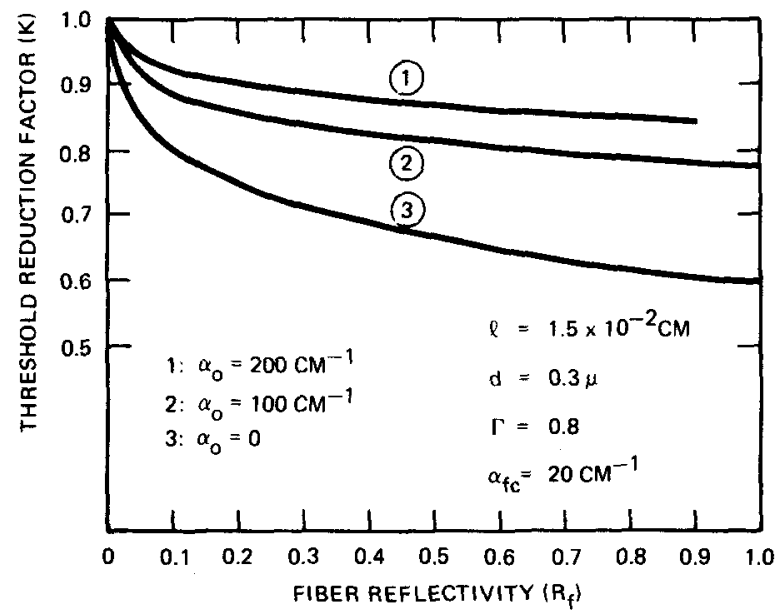

FIG. 4. Calculation of $J_{\mathrm{th} \mathrm{ER}} / J_{\mathrm{ih}}$ vs external reflectivity $\left(R_{f}\right)$. 
between the laser cleaved facet and the facet coating on the fiber end. Since the semiconductor laser is an oscillator, the phase delay $2 \delta$ will adjust itself such that $R_{\text {eff }}$ is maximized. Thus $R_{\text {eff }}$ becomes

$$
R_{\mathrm{eff}}=\left(R^{1 / 2}+R_{f}^{1 / 2}\right)^{2} /\left[1+\left(R_{f} R\right)^{1 / 2}\right]^{2},
$$

where $R_{f}$ is defined as the reflection from the external mirror and includes various loss mechanisms. In Fig. 4 we plot $K$ as a function of $R_{f}$ with $\alpha_{0}$ as a parameter. For the calculation we use $l=1.5 \times 10^{-2} \mathrm{~cm}, d=0.3 \mu, \Gamma=0.8, R=0.3$, and $\alpha_{\mathrm{fc}}=20 \mathrm{~cm}^{-1}$. These numbers were either measured or assumed to be typical numbers for the types of lasers used. A reasonable value for $\alpha_{0}$ is $100-200 \mathrm{~cm}^{-1} .{ }^{20}$ In earlier work $\alpha_{0}$ was set to equal zero. ${ }^{18,21}$ Using Fig. 4 we see that this assumption will tend to underestimate $R_{f}$ for a given $K$. Furthermore, the variation in the parameter $\alpha_{0}$ among different laser structures can help explain the different laser sensitivities to external feedback.

In order to compare the experimental value of $K$ with theory we need to estimate $R_{f}$. Unfortunately, a rigorous calculation is not possible. However, by making some simplifying assumptions we can make a crude estimate for $R_{f}$. The value of $R_{f}$ is then used to calculate $K$ and agreement with experiment is found to be satisfactory. We assume that only the fundamental mode is propagating (two polarizations are possible). The reflection coefficient for the fiber resonator can be estimated by assuming

$$
R_{f}=R_{\mathrm{Au}} \eta,
$$

where $R_{\mathrm{Au}}$ is the fraction of the light returned at the Aucoated end of the fiber and $\eta$ is a coupling coefficient given by

$$
\eta=\eta_{c} \eta_{\rho} \eta_{R}
$$

where $\eta_{c}$ is the input coupling coefficient at the lens, $\eta_{p}$ is the coupling coefficient due to depolarization effects, and $\eta_{R}$ is the coupling at the air-lens interfact. Since the length of the fibers used are typically less than $10 \mathrm{~cm}$, we neglect absorption losses. The input coupling coefficient is calculated by first calculating the half-acceptance angle $\theta_{h}$ for a spherical lens on a multimode step index fiber. The half-angle is ${ }^{15}$

$$
\begin{aligned}
\theta_{h}= & \sin ^{-1}\left\{n_{c} \sin \left[\sin ^{-1}(d / 2 r)+\cos ^{-1}\left(n_{c l} / n_{c}\right)\right]\right\} \\
& -\sin ^{-1}(d / 2 r),
\end{aligned}
$$

where $n_{c}$ and $n_{c 1}$ are the effective core and cladding index of refractions, $d$ is the diameter of the core, and $r$ is the radius of the fiber lens. In order to estimate $\theta_{h}$ for a graded index fiber we assume that the index vaariation can be represented by an equivalent step index. Using Eq. (11) and assuming $n_{c}=1.5$, $n_{c 1}=1.4935$ [these values are chosen to give the correct acceptance angle for $d / 2 r=0$ (flat fiber end), which is $\theta_{h}$ $=0.14], d=65 \mu$, and $r=110 \mu$, we find $\theta_{h}=0.3117 R$. The coupling efficiency $\eta_{c}$ into the fiber is estimated ${ }^{22}$ by integrating the farfield distribution, $I(\theta)$ of the laser over the acceptance angle of the fiber and normalizing by the total power emitted by the laser. We can write

$$
\eta_{c}=\int_{0}^{\theta_{h}} I(\theta) \sin \theta d \theta / \int_{0}^{\pi / 2} I(\theta) \sin \theta d \theta,
$$

where we have assumed that the beam divergence of the laser in the lateral direction is negligible and the distance between the laser and the fiber lens is sufficiently small such that the beam pattern falls within the fiber core cross section. The far-field distribution of the laser in the transverse plane can be approximated by

$$
\begin{aligned}
I(\theta) & =I_{0} \cos (2.4 \theta), & & \theta \leqslant 0.65 R \\
& =0, & & \theta \geqslant 0.65 \mathrm{R} .
\end{aligned}
$$

Using Eqs. (11) and (12) with $\theta_{h}=0.3771 R$ we find $\eta_{c}$ $=0.43$. One effect of the lens is to signficantly increase the coupling efficiency of the fiber resonator compared to a flat end. For example, when $d / 2 r=0$ (i.e., a flat end) $\eta_{c} \cong 0.10$. The maximum value of $\eta_{c}$ approaches $90-100 \%$, when $d / 2 r \cong 0.51$. Another effect of the lens is to provide beam refocusing into the active region of the laser. Since the lens is an integral part of the resonator, the tolerances in alignment can be relaxed compared to a discrete fiber and lens combination.

Another effect which must be considered is the polarization conversion in the fiber. ${ }^{23}$ The depolarization coupling coefficient $\eta_{p}$ is related to the rotation of the input polarization. Since the output of the injection laser is primarily polarized along the junction plane (TE polarization), the polarization conversion in the fiber effectively reduces the efficiency of the light coupling back into the laser. It has been observed that a length of $50 \mathrm{~cm}$ in a step-index multimode fiber randomizes the polarization of the incident wave. ${ }^{24}$ For a single-mode fiber, the fiber length required for depolarization is much larger $(>300 \mathrm{~m})$. We believe the polarization length for a graded index fiber is similar to that of a stepindex fiber. However, the results are a strong function of fiber stress and inhomogeneities. In our experiment there is a further complication due to the scattering from the evaporated fiber end reflector. Thus the depolarization is difficult to estimate. As a worst case estimate we will assume that the TE depolarization coupling coefficient $\eta_{p}=0.5$.

Finally, the coupling coefficient $\eta_{R}$ is related to the reflection loss at the air-fiber interface and is

$$
\eta_{R}=1-\left(\left(\eta_{c}-1\right) /\left(\eta_{c}+1\right)\right)^{2} \text {. }
$$

Using Eqs. (11), (13), (14), and $R_{\mathrm{Au}}=1$, we find $R_{f}=0.2$. Using Fig. 4 we find $0.86<K<0.91$ (for $100<\alpha_{0}<200$ ). The experimental value of $K$ is 0.92 (obtained from Fig. 3).

We find fairly good agreement considering the crudeness of our calculations. We should note that better agreement can be obtained by assuming $0.1<R_{f}<0.2$ instead of using the calculated value of $R_{f}=0.2$. An improvement in $R_{f}$ can be obtained by using a fiber lens with a smaller radius of curvature (for, e.g., $r \sim 50 \mu$ ) to improve the input coupling. Furthermore, our calculation has assumed singlemode propagation in the fiber. This assumption is quite poor and will lead to discrepancies between measurement and calculation. If one uses a single-mode fiber to ensure singlemode propagation, then a lens radius $\sim 10-20 \mu$ is required for efficient coupling. ${ }^{25}$ One could expect maximum theoretical coupling efficiencies ranging from $30-90 \%,{ }^{25} \mathrm{de}-$ pending on the near-field width and beam ellipticity. One serious drawback in using a single-mode fiber is the rigid alignment tolerances required. 


\section{SELF-LOCKING EFFECTS IN INJECTION LASERS OPERATING IN AN EXTERNAL CAVITY: THEORETICAL CONSIDERATIONS}

The frequency response of injection lasers operating in an external cavity has been discussed by several authors. Broom et al. ${ }^{12}$ observed a sharp resonance in the frequency response when the inverse transit time or a harmonic was equal to the relaxation oscillation frequency. The results were explained by assuming an interaction between photon and injected carriers and longitudinal-mode coupling effects. When a small sinusoidal input at the resonance frequency was applied, $100 \%$ modulation of the light output was obtained. No sharp pulsations of the light were observed. Instead the light output was almost sinusoidal. In recent experiments a sharp pulsation was observed when the laser was driven at the resonance.$^{8-10}$ Pulses as short as $20 \mathrm{ps}$ at $3-\mathrm{GHz}$ repetition rates have been reported. Other experiments have used an external cavity to reduce the amplitude of relaxation oscillations in pulsed operation. ${ }^{26}$ Lastly, an external cavity has been used to lock the frequency of selfpulsing lasers." The locking action can lead to either an enhancement ${ }^{11}$ or a suppression ${ }^{13,14}$ of the pulsation amplitude depending on the external cavity length. Many of the above results are not adequately explained by the conventional rate equations, which do not predict sustained resonances or pulsations. In recent publications ${ }^{14,27}$ we have shown that an external cavity can significantly alter the characteristics of self-pulsations. Suppression of self-pulsations was predicted and observed if the external cavity length was properly chosen. The model consists of the conventional rate equations used to analyze relaxation oscillations in injection lasers with the addition, in the manner of Copeland, ${ }^{28}$ of absorbing electron traps, and also by a term accounting for the feedback due to the external resonator. By using these equations we find that many of the observations previously mentioned are not independent, and fit well within the scope of a single model. We should further note that by using the rate equation approach we have inherently neglected coherence effects. A rigorous treatment which considers coherence of the fields is very difficult and is beyond the scope of this paper. Our calculations are compared to experimental results on lasers operating with an external fiber and a conventional external resonator. This is discussed in Sec. IV. Although we use Copeland's equations in our calculations, we believe that other well-known models with saturable absorbers ${ }^{29-31}$ or superlinear gain, ${ }^{32,33}$ when modified to include the external cavity, would lead to similar results. Our starting point is the equations given by Copeland. ${ }^{27}$

$$
\begin{aligned}
\frac{d N_{e}(t)}{d t}=\frac{J}{e d} & -\frac{N_{e}(t)}{\tau_{s}}-A N_{\mathrm{ph}}(t)\left[N_{e}(t)-N_{0}\right] \\
& +\frac{d T(t)}{d T} \\
\frac{d N_{\mathrm{ph}}(t)}{d t}= & \frac{-N_{\mathrm{ph}}(t)}{\tau_{\mathrm{ph}}}+A N_{\mathrm{ph}}\left[N_{e}(t)-N_{0}\right] \\
& +N_{e}(t) / \tau_{s}-\sigma_{0} c_{0} N_{\mathrm{ph}}(t)\left(T_{0}-T\right) \\
& +\left(R_{f} N_{\mathrm{ph}} / \tau_{\mathrm{ph}}^{\prime}\right)(t-\tau)
\end{aligned}
$$

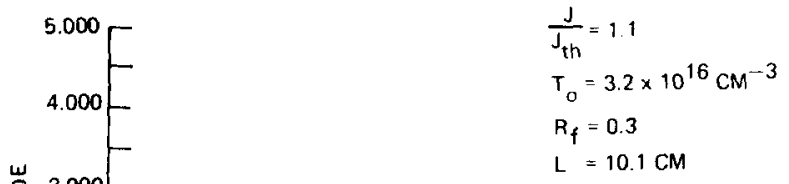

(a)

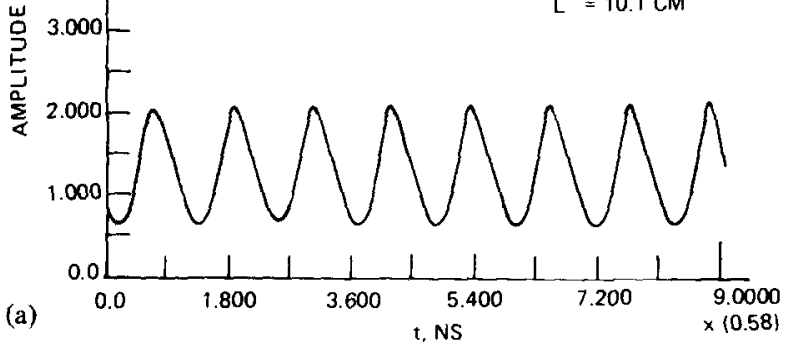

(b)

$$
\begin{aligned}
& \frac{J}{J}=1.1 \\
& T_{0}=4.13 \times 10^{16} \mathrm{~cm}^{-3} \\
& R_{f}=0.3
\end{aligned}
$$

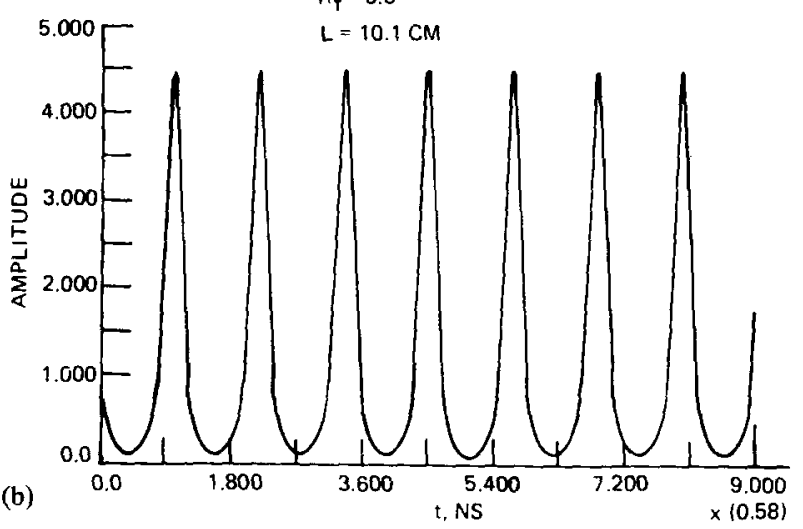

FIG. 5. Calculation of the temporal display of an injection laser-external resonator configuration displaying pulsations. (a) $T_{0}=3.2 \times 10^{16} / \mathrm{cm}^{3}$; (b) $T_{0}=4.13 \times 10^{16} / \mathrm{cm}^{3}$. In both cases the laser does not pulse without the external cavity.

$$
\frac{d T(t)}{d t}=\sigma_{0} c_{0} N_{\mathrm{ph}}(t)\left[T_{0}-T(t)\right]-\sigma_{e} v N_{e}(t) T(t) .
$$

The first two equations with the exception of the last terms (those involving $T$ and $R^{\prime}$ ) are the conventional rate equations, with $N_{e}\left(\mathrm{~cm}^{-3}\right)$ being the electron density and $N_{\mathrm{ph}}\left(\mathrm{cm}^{-3}\right)$ the photon density. In these equations $J$ is the current density $\left(\mathrm{A} / \mathrm{cm}^{2}\right), e(C)$ is the electronic charge, and $d(\mathrm{~cm})$ is the active region thickness. The effect of transverse optical confinement and current spreading are neglected.

The term $\left(R^{\prime} N_{\mathrm{ph}} / \tau_{\mathrm{ph}}^{\prime}\right)(t-\tau)$ represents the delayed feedback from the external mirror, where $\tau$ represents the round trip time in the external cavity and is given by $\tau=2 n_{c} L / c_{0}$, where $n_{c}$ is the index or refraction in the resonator, $L$ is the external cavity length, and $c_{0}$ is the speed of light in vacuum; $\tau_{\mathrm{ph}}^{\prime}$ is the photon lifetime related to the mirror loss only and is given by $\tau_{\mathrm{ph}}^{\prime}=\left(c_{0} / n l\right) \ln (1 / R)$, where $n$ is the index of refraction for GaAs and $l$ and $R$ have been previously defined in Eq. (6); $R_{f}$ is the amount of light fed back into the laser. The last equation represents the equation of motion for the traps, where $c_{0}$ is the speed of light in the medium, $T$ is the density of empty traps, $T_{0}$ is the total number of traps, $\sigma_{e}$ is the electron capture cross section, $v$ is the thermal velocity, and $\sigma_{0}$ is the photon capture cross section by a trap occupied by an electron (whose density is $T_{0}-T$ ). For the calcula- 
tions we use $A=1.5 \times 10^{-6} \mathrm{~cm}^{3} / \mathrm{s}, \tau_{s}=1.5 \times 10^{-9} \mathrm{~s}, \tau_{\mathrm{ph}}$ $=1.4 \times 10^{-12} \mathrm{~s}, d=1 \times 10^{-4} \mathrm{~cm}, N_{0}=5 \times 10^{17} \mathrm{~cm}^{-3}$, $\beta=10^{-4}, c_{0}=8 \times 10^{9} \mathrm{~cm} / \mathrm{s}, \sigma_{e}=1.5 \times 10^{-17} \mathrm{~cm}^{2}$, $V=4.42 \times 10^{7} \mathrm{~cm} / \mathrm{s}$, and $\sigma_{0}=3 \times 10^{-16} \mathrm{~cm}^{2}$. The numbers used are reasonable values and can be obtained from Refs. 22 and 28. The parameter $\left(\sigma_{0} T_{0}\right)$ in the model determines the degree of pulsation. This parameter is related to the strength of the superlinear gain and saturable absorption. The calculations were performed by integrating Eqs. (15)-(17) using the Runge Kutta fourth-order algorithm. The system is excited with a step change in current and run until steady-state oscillation or its absence is confirmed. In Figs. 5(a) and 5(b) we show a plot of the light output as a function of time for lasers with $T_{0}=(3.2$ and 4.13$) \times 10^{16} \mathrm{~cm}^{-3}$, respectively. The lasers are operated in an external cavity with $R_{f}=0.3$. In both cases the lasers do not pulse when the external cavity is not present. The calculated result indicates that induced pulsations can be obtained if an external cavity is present. The degree of pulsation depends on the actual trap density. In Fig. 5(a) the output is similar to a sinusoidal, while in Fig. 5(b) the output resembles a pulsation. The difference in trap density is $\sim 0.9 \times 10^{16} \mathrm{~cm}^{-3}$, indicating that only a small difference in the density of absorbers can affect the pulse shape. These results can help explain the sharp contrast between the result of Broom et al. ${ }^{12}$ and other workers. ${ }^{8-10}$ The effect described above is very similar to passive mode locking when operating a laser-saturable absorber combination. ${ }^{7} \mathrm{We}$ would expect much shorter optical pulses using this mechanism compared to active mode locking. In Fig. 6 we show a plot of the amplitude of the induced pulsation versus external cavity length. The plots are taken for the same parameters as previously discussed; however, the results do not change significantly for $0.2<R_{f}<0.3$. The results are very similar to the observations by Broom et al. ${ }^{12}$ One should also note the frequency jumping phenomenon has been previously observed, ${ }^{11}$ but never fully explained. By applying an rf signal with $10 \%$ modulation depth (10\% of $\left[I-I_{\mathrm{th}}\right]$ ) to the laser (with a frequency corresponding to $f_{R}$ or a harmonic) it is possible to narrow the pulsewidth by $50 \%$.

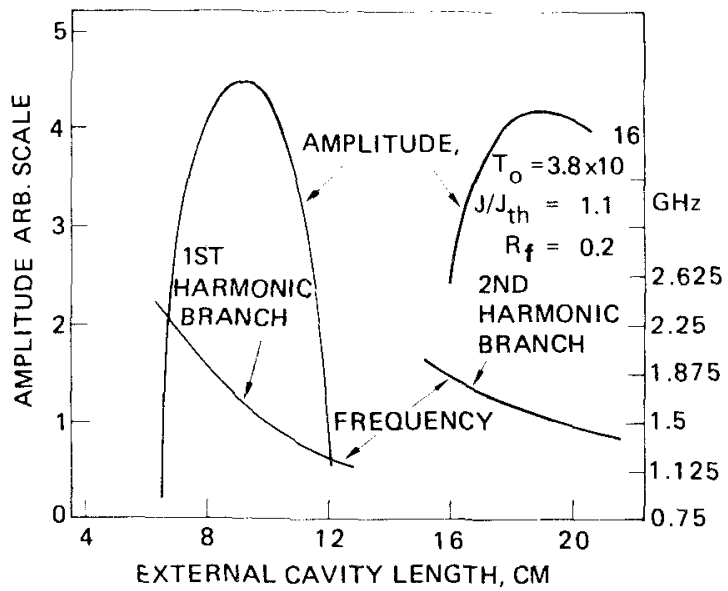

FIG. 6. Calculation showing the amplitude of the induced pulsations vs external cavity length.

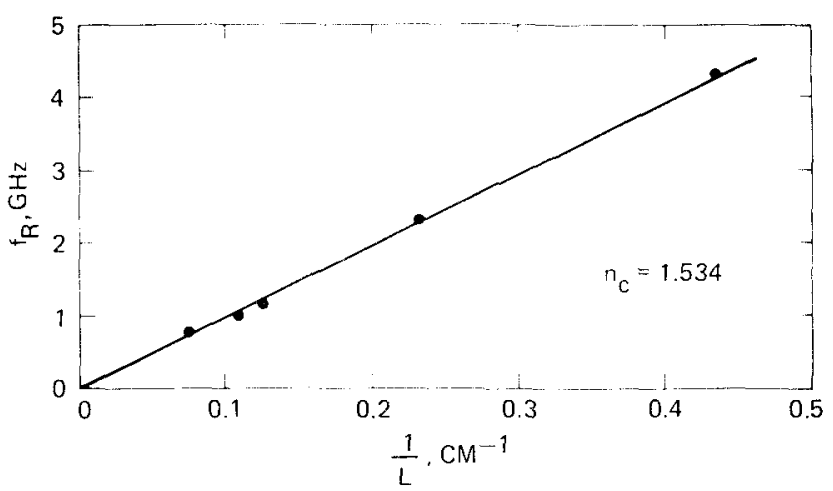

FIG. 7. Round trip resonance frequency $\left(f_{r}\right)$ vs $1 / L$ (where $L$ is the external cavity length).

\section{EXPERIMENTAL RESULTS: FREQUENCY AND TEMPORAL CHARACTERISTICS}

In this section we discuss our experimental results on the frequency and temporal characteristics of injection lasers operating in an optical fiber external resonator. In our studies we have observed in some cases results similar to those reported by Broom et al. ${ }^{12}$ However, in other cases we observed a spiking of the light output at the cavity transit time or a harmonic. Using the model developed in the pre-
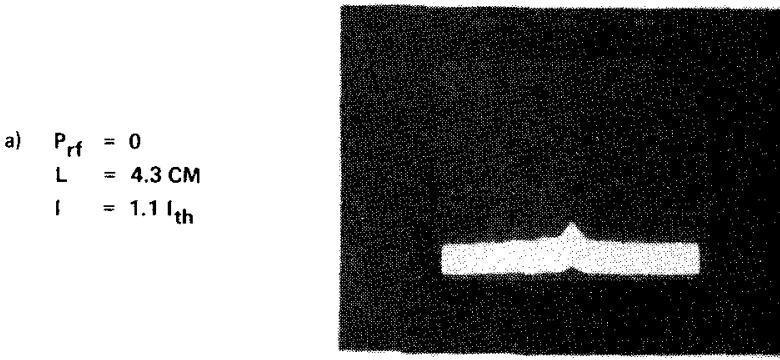

b)
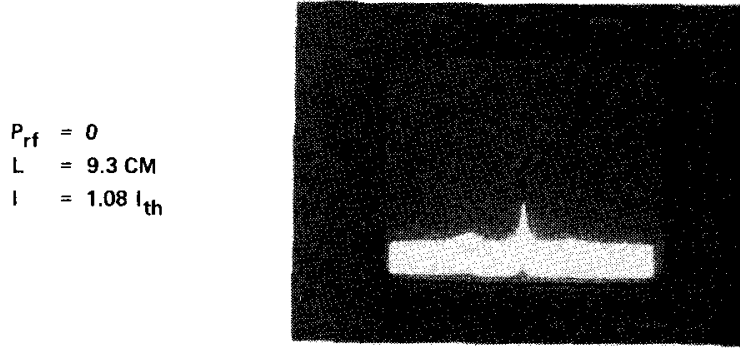

$$
\begin{aligned}
P_{\text {rf }} & \approx 1-2 \mathrm{~mW} \\
L & =9.3 \mathrm{cM} \\
\mathrm{I} & =1.08 \mathrm{I}_{\mathrm{th}}
\end{aligned}
$$

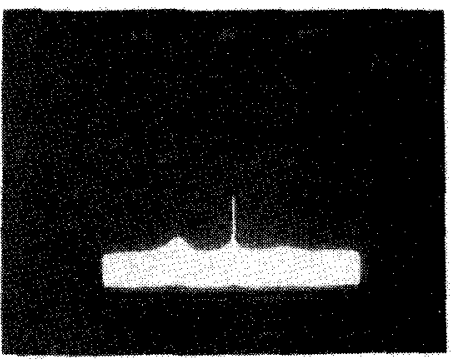

FIG. 8. (a) Microwave spectrum of the light output for a typical laser with an optical fiber resonator. (b) Microwave spectrum in some lasers operating with an optical fiber resonator. Note the sharper resonance compared to (a) and operation approximately at $2 f_{r}$. (c) Same as (b) except we apply an if signal at the resonance. Note the narrowing of the spectrum. 


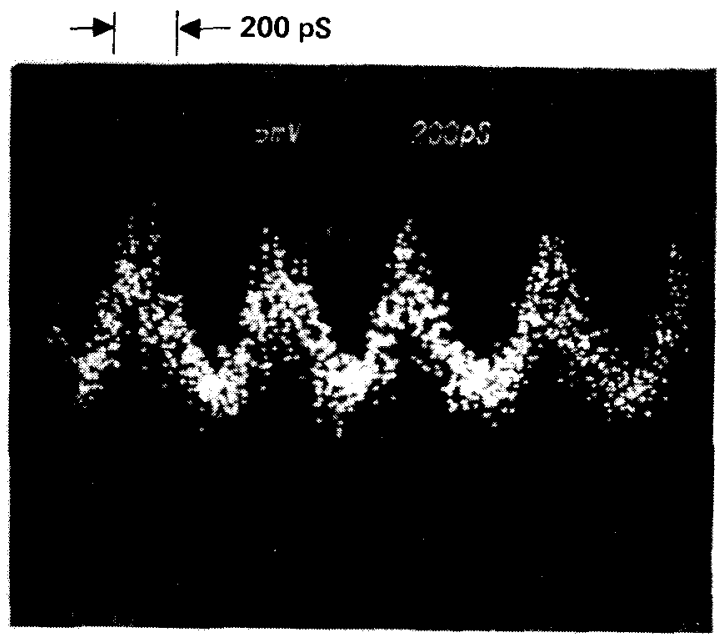

a)

(POSITIVE GOING)

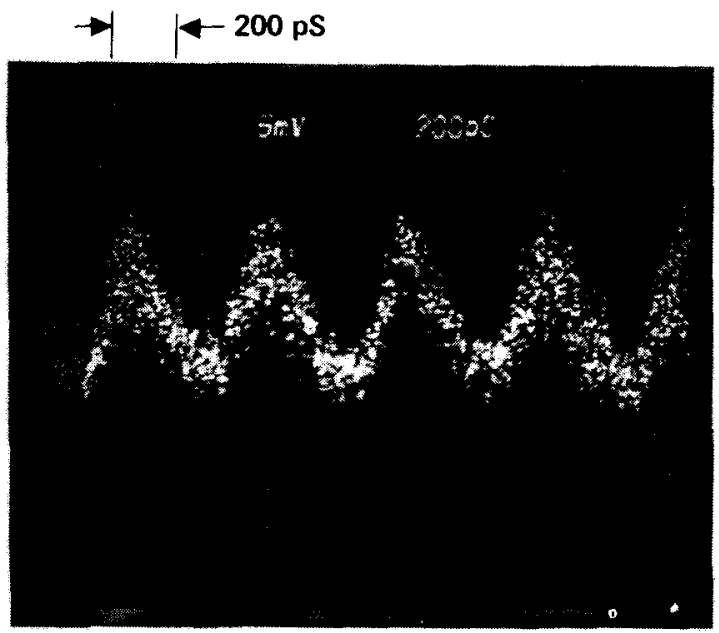

b)

(POSITIVE GOING)

FIG. 9. (a) Output pulse when we apply a sinusoidal rf signal at the resonance frequencies. The output corresponds to the laser in Fig. 8(a). (b) Same as (a) except we drive the laser at $\frac{1}{2} f_{R}$.

vious section we are able to qualitatively explain our results. In Fig. 7 we plot the cavity resonance frequency $f_{R}$ as measured using the spectrum analyzer versus fiber length. The resonance observed is similar to that reported by Ho et al. ${ }^{10}$ The frequency is generally related to the fiber length by

$$
f_{R}=c / 2 n_{c} L=1 / \tau,
$$

where $n_{c}$ is the effective index of the fiber core. From Fig. 7 we find $n_{c}=1.53$, which is in reasonable agreement with the expected values $n_{c}=1.5$. A typical light output versus frequency is shown in Fig. 8. The width of the resonance is typically $25-50 \mathrm{MHz}$ when the rf powser is zero. However, in some cases, as shown in Fig. 8(b), the resonance can be quite narrow. In this case the half-width is less than $10 \mathrm{MHz}$. We should note that we have observed a similar effect in other lasers. ${ }^{34}$ In Fig. 9 we show temporal displays of the light output [corresponding to Fig. 8(a)] when we apply modulation current at two different input frequencies $\left(f_{R}\right.$ and $\left.\frac{1}{2} f_{R}\right)$. The output is modulated, but the pulse does not

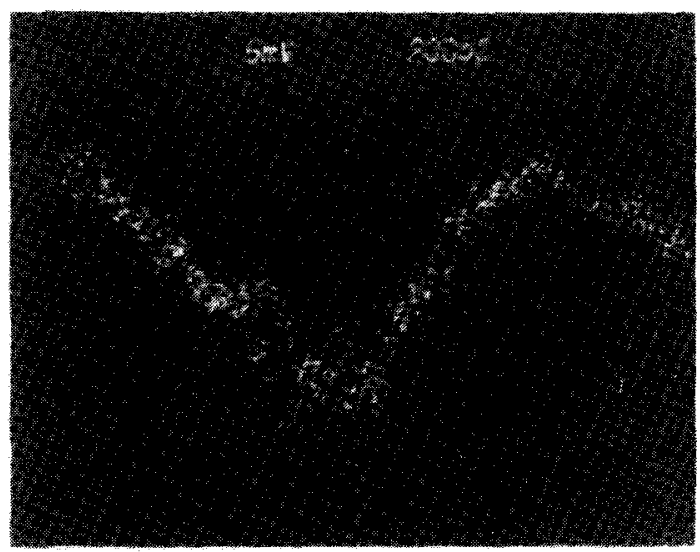

a) NO ALIGNMENT

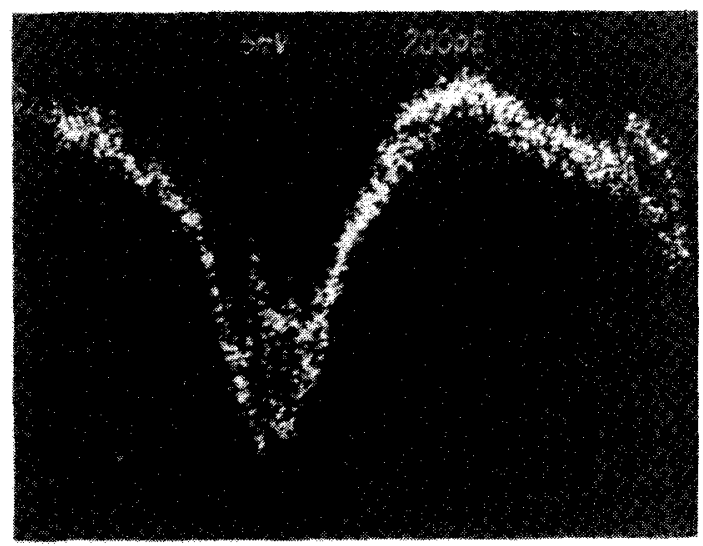

b) ALIGNMENT

FIG. 10. Same as Fig. 9 except we increase the length of the external fiber resonator $(L=9.3 \mathrm{~cm})$. Note the sharper rise time of the pulse when the fiber is aligned. The laser is driven at $f_{R}$ and the $\mathrm{rf}$ power is $\sim 1-2 \mathrm{~mW}$.

appear extremely sharp, with pulsewidths greater than 200 ps. One possible reason for the broadening is the instability of the resonance of $f_{R}$. We have observed a significant

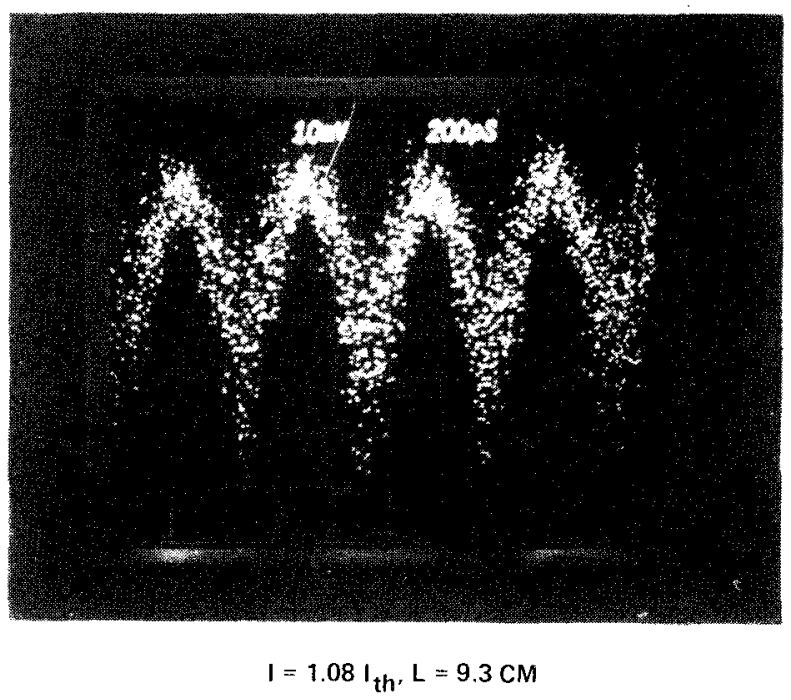

FIG. 11. Output pulse shape for a laser-external fiber resonator combination. The laser is the same as in Fig. 8(b). The laser is driven at $2 f_{K}$ and $\mathrm{rf}$ power is $\sim 1-2 \mathrm{~mW}$. Note the relatively sharper pulsations compared to Fig. 9. 


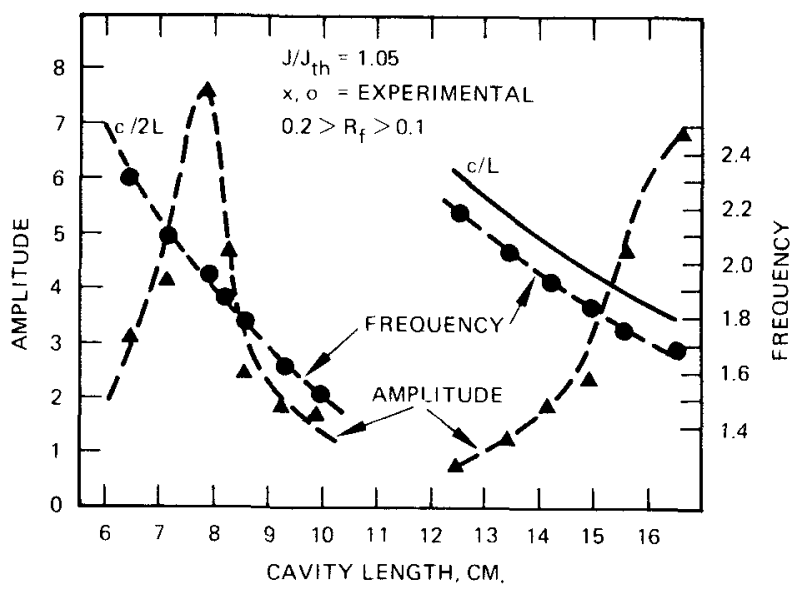

FIG. 12. Experimental amplitude and frequency of induced resonance vs external cavity length.

amount of frequency jitter. Another reason is the APD used which has a maximum 3-dB cutoff frequency estimated to be $2.65 \mathrm{GHz}$. A measurement of the second-harmonic laser output at $4.58 \mathrm{GHz}$ was $13 \mathrm{~dB}$ below the fundamental. Misa-

\section{NO EXTERNAL CAVITY $I=1.07 I_{\text {th }}$}

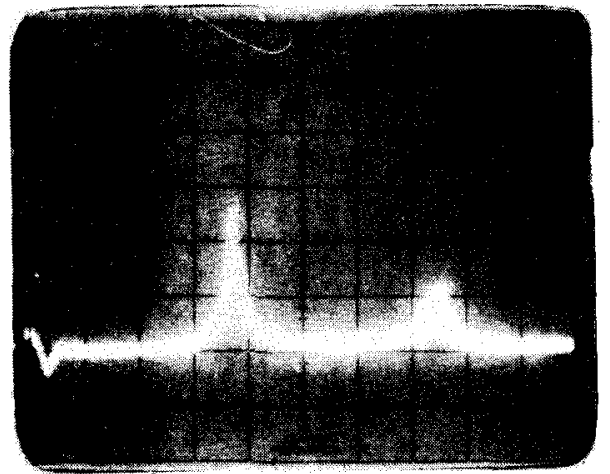

VERT: $10 \mathrm{~dB} / \mathrm{DIV}$

HORZ: $180 \mathrm{MHz} / \mathrm{DIV}$

\section{EXTERNAL CAVITY} (EFR)

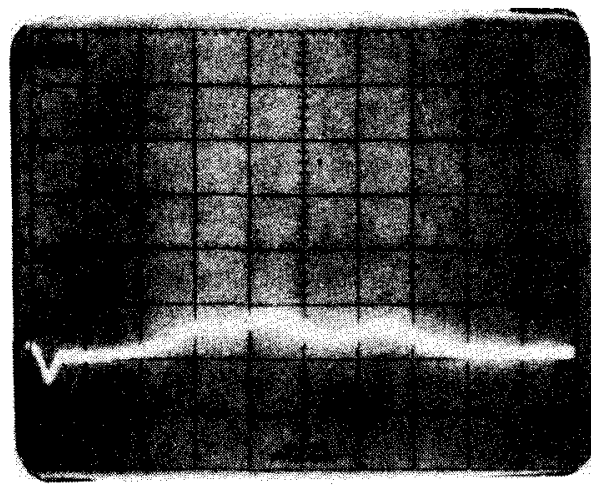

$$
\begin{aligned}
& I=1.07 I_{\text {th }} \\
& L=9.3 \mathrm{~cm}
\end{aligned}
$$

FIG. 13. (a) Photograph displaying self-pulsations with no external cavity. (b) Suppression of seif-pulsations by aligning an optical fiber resonator.
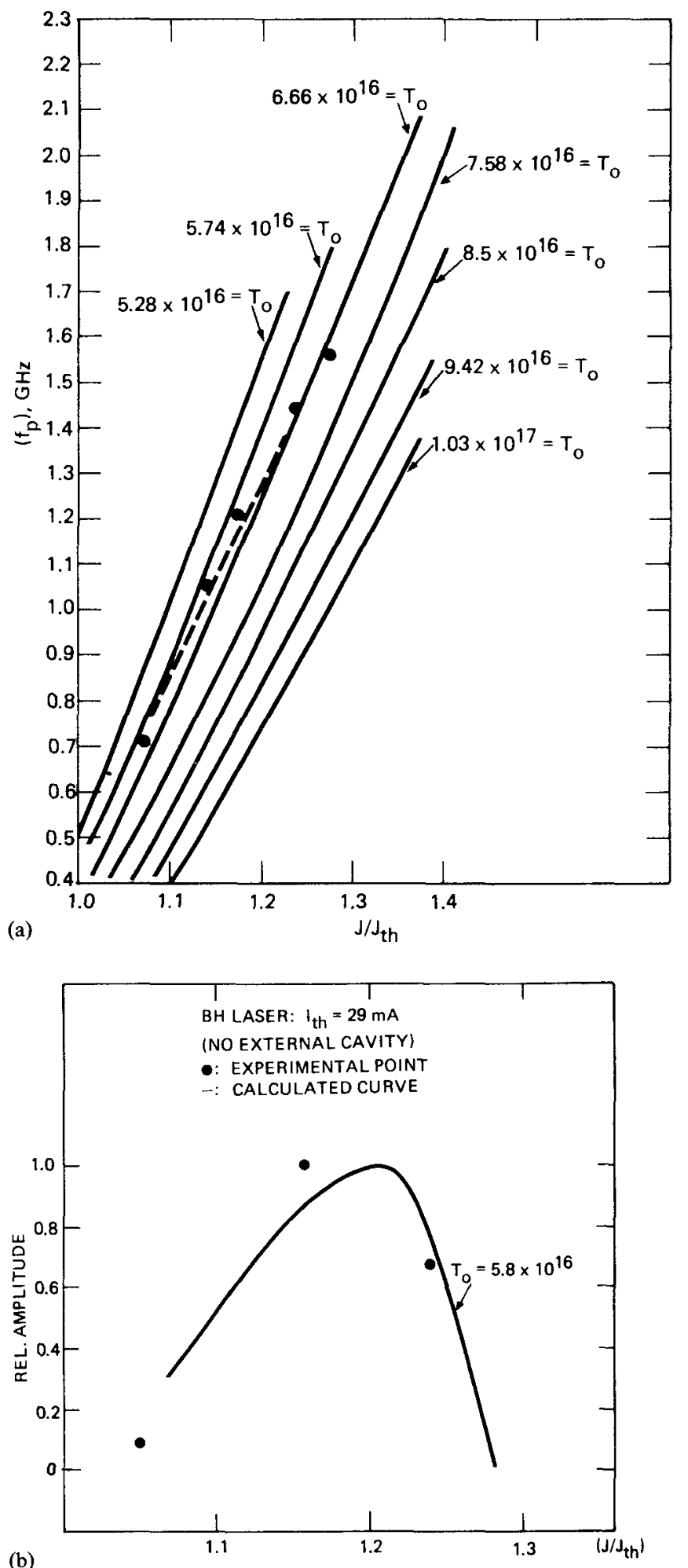

FIG. 14. (a) Calculations displaying frequency of self-pulsation (with no external cavity) vs injection current. The dots represent experimental results. (b) Calculation of the amplitude of self-pulsations vs injection current.

lignment of the external cavity produces a second-harmonic signal which is at least $20 \mathrm{~dB}$ below the fundamental. It is evident from Fig. 9 that the resonance at $f_{R}$ can be exited by driving the laser at $f_{R}$ or $\frac{1}{2} f_{R}$. This form of subharmonic locking has been observed in most of the laser-external resonator combinations tested. We have observed that as the 


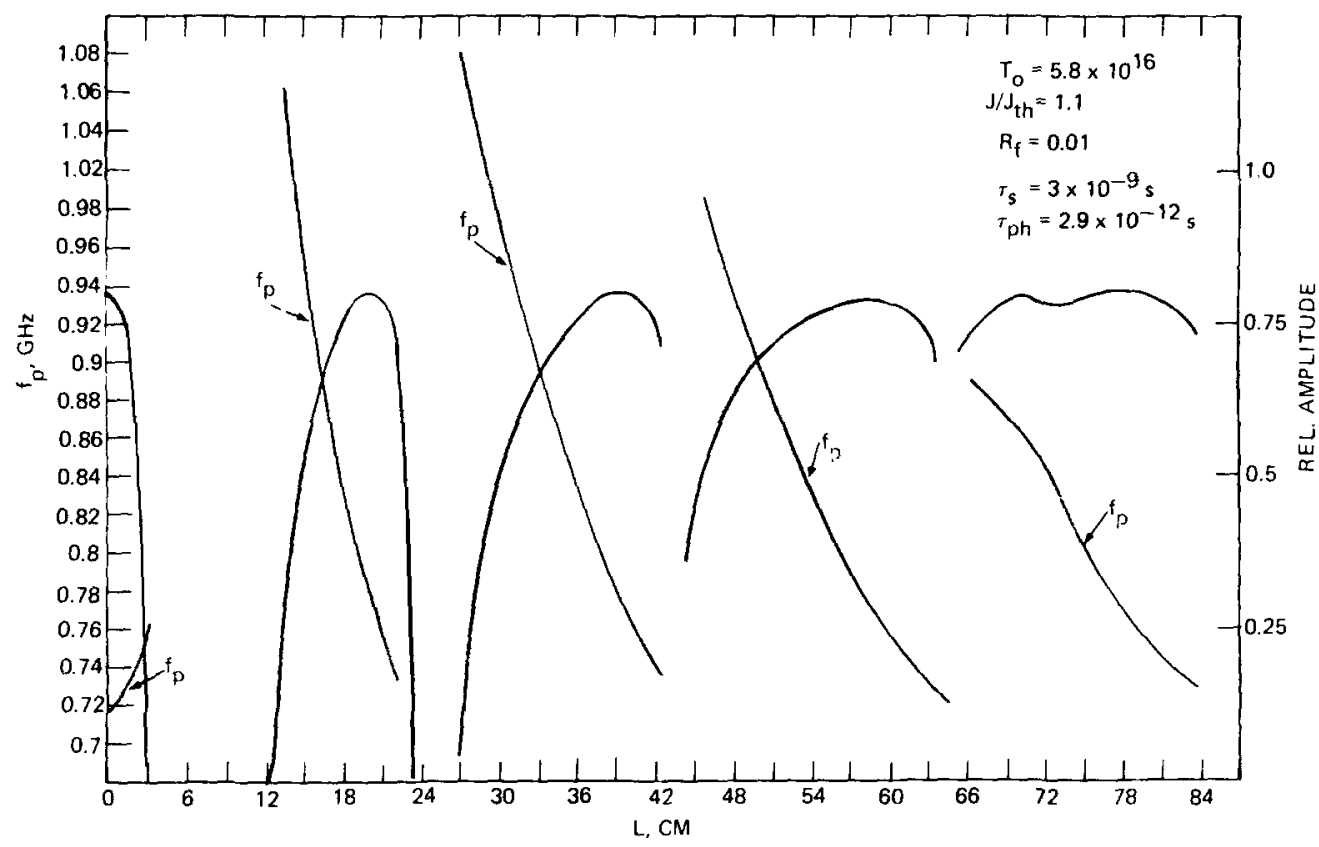

FIG. 15. Calculated plot of the amplitude and frequency vs external cavity length for a self-pulsing laser.

drive frequency approaches $\frac{1}{2} f_{R}$, the light outut at $f_{R}$ and $2 f_{R}$ increase significantly, while the power at $\frac{1}{2} f_{R}$ decreases. The bandwidth of this interaction is similar to the bandwidth of the resonance. No enhancement effect is observed when the (EFR) is misaligned.

In Fig. 10 we show the light output for the same laser shown in Fig. 8(a), except the fiber resonator has been lengthened. We note a significant narrowing of the pulse output when the fiber resonator is aligned. In both the aligned and misaligned cases the output power is adjusted to give approximately the same modulation depth. The sharper pulse shape compared to Fig. 9 may arise from the resonant behavior of the self-pulsations as shown in Fig. 6. Thus we conclude that in order to generate shorter pulses the external cavity length must be properly chosen.

In Fig. 11 we show a photograph of a pulsation occurring at $2 f_{R}$ using the laser with frequency response displayed in Fig. 8(b). When the laser is driven sinusoidally, the light output consists of pulses $\sim 150 \mathrm{ps}$ in width and the measurements are detector limited. These results are qualitatively explained by the model presented in Fig. 6. Depending on the external cavity length the pulsation occurs at a harmonic of $f_{R}$. Comparing Figs. 9-11 we note the results are very similar to the calculated results shown in Figs 5 and 6. It appears that differences in the output characteristics of pulsing lasers can be qualitatively explained by the difference in the degree of saturable absorption strength $\sigma_{0} T_{0}$ and the length of the external optical cavity. In order to further correlate this assumption we have performed an experiment on the laser described in Fig. 11. The experiment consists of using a conventional external cavity composed of a microscope objective and a flat mirror. In Fig. 12 we plot the amplitude of the induced resonance (as measured on a spectrum analyzer) versus external cavity length. Note the resonant behavior and the frequency jumping phenomena. These results are surprisingly similar to those predicted in Fig. 6 thus giving further support to our model. Further experiments are described in Ref. 27.

In Fig. 13 we demonstrate the use of an EFR to suppress self-pulsations. For the experiment, a self-pulsing laser is used $\left(I_{\mathrm{th}} \sim 29 \mathrm{~mA}\right)$. A plot of the frequency and the amplitude of the pulsation (without an external cavity) versus injection current is shown in Fig. 14. A good fit to the experimental data can be obtained for $T_{0} \sim 5.8 \times 10^{16} \mathrm{~cm}^{-3}$, as can be obtained from Fig. 14. In the case of a pulsing laser, $T_{0}$ is such that the isolated laser pulses without an external cavity. The effect of an external cavity is to either enhance or suppress intensity pulsations depending on the external cavity length. A calculation showing amplitude of pulsations versus external cavity length is shown in Fig. 15. Details have been previously described in Ref. 14. For our purposes here, we note the first suppression band occurs for $3<L<12 \mathrm{~cm}$. Experimentally, we have obtained suppression of pulsations by using a conventional external cavity and by using a fiber resonator with $L \sim 9.3 \mathrm{~cm}$. The case of the fiber resonator is shown in Fig. 13 which give amplitude of pulsations versus frequency as shown on a spectrum analyzer. A closer look shows the amplitude of the puisation is reduced by $\sim 20 \mathrm{~dB}$ and the resonance is broadened significantly. The suppression of the self-pulsation is maintained for currents up to 1.1 $I_{\mathrm{th}}$.

Lastly, we would like to discuss our experimental results on using a short electrical pulse synchronous with the cavity resonance. The technique of using a short-pulse excitation in order to mode lock a laser has been used in the mode locking of dye lasers by synchronously pumping with an $\mathrm{Ar}^{+}$laser. ${ }^{35}$ The technique consists of exciting the dye with a 100-ps pulse and producing ultrashort (a few ps) pulses from the dye laser. In our experiments we have used a short electrical pulse in order to generate short optical pulses at 1$\mathrm{GHz}$ repetition rates. The short electrical pulses are generated by using an HP step-recovery diode with a $1-\mathrm{GHz}$ center 


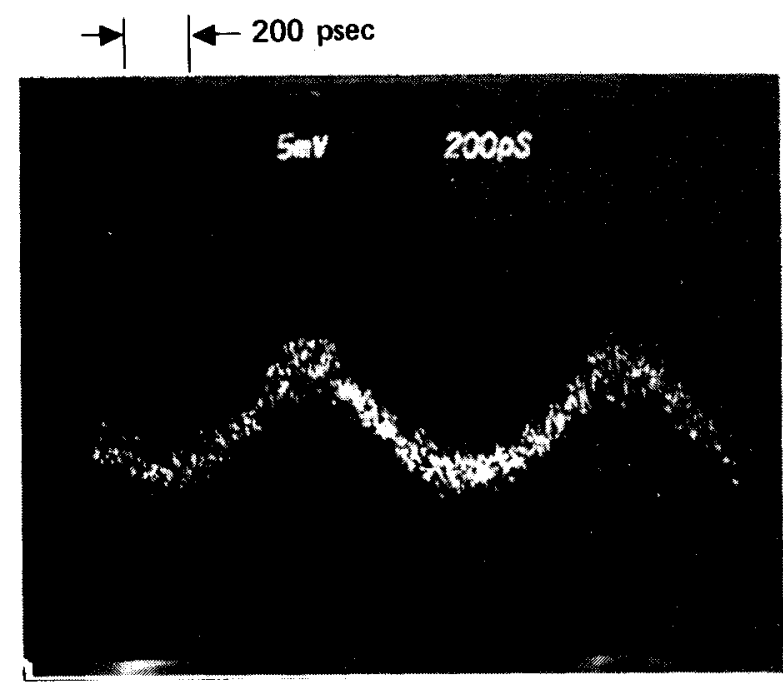

(a) No alignment.

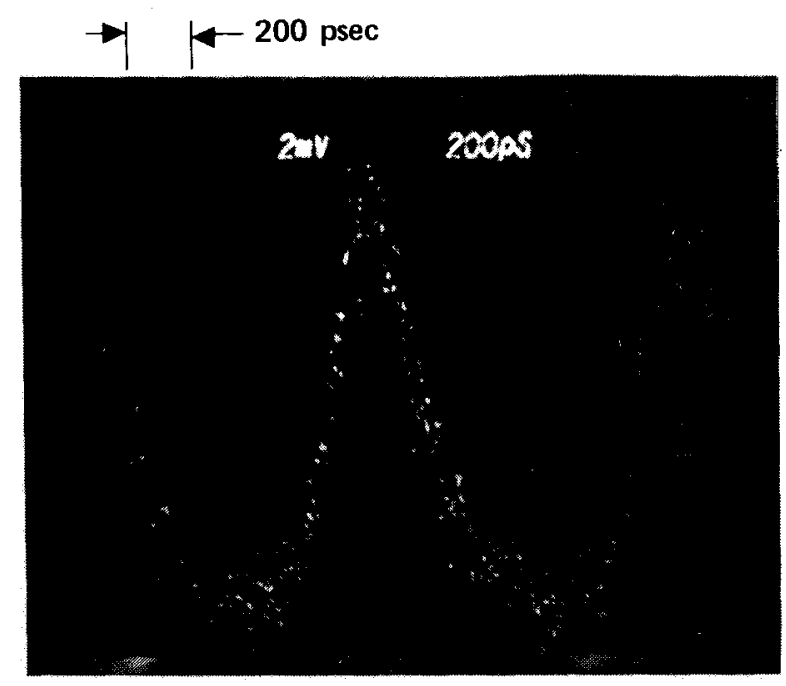

(b) With alignment.

FIG. 16. Temporal display of synchronously pumped injection laser (the laser is similar to that in Fig. 8(a)). The laser is driven at $f_{R}(L=9.3 \mathrm{~cm})$.

frequency and a useful bandwidth of $200 \mathrm{MHz}$. These diodes can provide useful harmonic power up to $18 \mathrm{GHz}$, and thus should provide a very efficient scheme for synchronously mode locking an injection laser. Even though the step recovery diode can provide a broad bandwidth, the laser and package combination tends to reflect a large percentage of the input power for frequencies above $3.5 \mathrm{GHz}$. This effect should reduce the usefulness of this scheme unless a broad bandwidth input circuit is used.

The experimental arrangement is essentially the same as previously discussed except the step-recovery diode is placed in series with the rf input to the laser. In Fig. 16 we show a temporal display of the light output. The length of the fiber was approximately $9.3 \mathrm{~cm}\left(f_{R}=1.1 \mathrm{GHz}\right)$. Figure 16(a) shows the case when the EFR is not aligned while in Fig. 16(b) we show the case where the EFR is aligned. In both cases the rf input and the dc output power are approximately the same. We note that the pulse output is narrowed when the EFR is aligned.

\section{SUMMARY AND DISCUSSION}

In the previous sections we have discussed the characteristics of an optical fiber resonator in conjunction with (GaAl)As lasers. We find that the fiber resonator can be made mechanically rugged, light weight, and relatively easy to align. By proper alignment the threshold current of the laser is reduced 6-10\% and by modulating the injection current at the inverse round trip time $\left(f_{R}\right)$ we are able to generate pulses 150 ps wide at $\mathrm{GHz}$ rates. It is also possible to suppress self-pulsations in injection lasers by using an optical fiber resonator of the proper length. Our experimental results can be explained in terms of the conventional rate equations with the addition of saturable traps and a term accounting for the external feedback. The model can predict many of the previously unexplained phenomena such as selfsustained resonances, enhancement or suppression of selfpulsations, induced self-pulsations, frequency jumping, and mode locking. Even though our calculations use a trap model we feel that other models with similar mathematical formulations will predict similar results.

The results presented provide a method for diagnosing self-pulsations before they develop. Using the equations presented in Sec. III [Eqs. (15)-(17)] we can correlate the amplitude and bandwidth of the induced resonance versus external cavity length, with absorber density $T_{0}$. The experimental curves can be fitted to the calculations to give absorber density $T_{0}$. The test can be performed in a laser which does not self-pulse initially, but still contains a high density of absorbers. When this laser is placed in an external cavity, an induced resonance can be obtained. By monitoring the amplitude and bandwidth of the induced resonance versus time one can determine the time rate of change of the absorbers (i.e., $d T_{0} / d t$ ). It should then be possible to correlate laser structures and fabricational processes to $d T_{0} / d t$. The important concept is that these measurements can be made before the isolated laser begins to self-pulse. This technique should provide a convenient and nondestructive test.

In closing we would like to mention several passing thoughts. First, we have used a model for describing pulsations, which is mathematically similar to the description of a saturable absorber. It is important to point out that no physical basis for the model has been established. More experimental and theoretical work is clearly needed. Second, in the experiments we have used a multimode graded index fiber resonator. Surprisingly, our results are similar to those obtained by using a conventional external cavity. Furthermore, our analysis gives good agreement considering the crudeness of our approximations. It may be possible that we are observing some self-imaging properties of a quadratic index fiber. Lastly, it is conceivable that by using a single-mode fiber our results can be extended to long resonators. This can enable an almost continuously tunable pulse repetition rate by exciting the laser at a harmonic of $f_{R}$. 


\section{ACKNOWLEDGMENTS}

The authors are very grateful to $D$. Botez of RCA Research Labs., Princeton, N.J. for providing some of the lasers used. We also wish to thank T. R. Ranganath for useful discussions and a critical reading of the manuscript and $O$. G. Ramer for providing the fibers used. Lastly, we wish to thank R. Jain, C. Slayman, and G. L. Tangonan for discussions and D. L. Lewis for valuable experimental assistance. This work was partially supported by DARPA and monitored by NRL under Contract N00173-78-C-0192, NSF Grant ENG 7604927-A04, and ONR Contract NOO014-76C-0322.

'E. P. Ippen and C. V. Shank, Appl. Phys. Lett. 27, 488-490 (1975).

${ }^{2}$ H. Taylor, IEEE J. Quantum Electron. 15, 210 (1979).

${ }^{3}$ H. Yen, M. K. Barnoski, R. G. Hunsperger, and R. T. Melville, Appl. Phys. Lett. 31, 120 (1977).

${ }^{4} \mathrm{H}$. Yen and L. Figueroa, "Optical-Microwave Interactions in Semiconductor Devices," Hughes Research Laboratories report under contract N00173-78-C-0192, Sept. 1978.

${ }^{5}$ L. E. Hargrove, R. L. Fork, and M. A. Pollack, Appl. Phys. Lett. 5, 780 (1963).

${ }^{6}$ A. J. DeMaria, D. A. Stetser, and H. Heynan, Appl. Phys. Lett. 8, 174 (1966).

${ }^{7}$ A. Dienes, E. P. Ippen, and C. V. Shank, Appl. Phys. Lett. 19, 258 (1971).

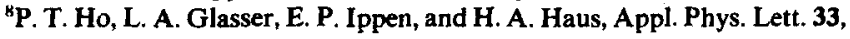
241 (1978)

${ }^{9}$ L. A. Glasser, Electron. Lett. 14, 725 (1978).

${ }^{10} \mathrm{P}$. T. Ho, Electron. Lett. 15, $526(1979)$.
'T.L. Paoli and J. E. Ripper, IEEE J. Quantum Electron. 6, 335 (1970).

${ }^{12}$ R. F. Broom, E. Mohn, C. Risch, and R. Salathe, IEEE J. Quantum Electron. 6, 335 (1970).

${ }^{13}$ N. Chinone, K. Aiki, and R. Ito, Appl. Phys. Lett. 33, 990 (1978).

${ }^{14}$ L. Figueroa, K. Y. Lau and A. Yariv, Appl. Phys. Lett. 36, 248 (1980).

${ }^{15} \mathrm{D}$. Botez, IEEE International Semiconductor Laser Conference, San Francisco, California, Oct. 1978, Paper C.3.

${ }^{16}$ D. Botez, Appl. Phys. Lett. 33, 872 (1978).

${ }^{17}$ D. Kato, J. Appl. Phys. 44, 2756 (1973).

${ }^{18}$ E. Mohn, 1968 Symposium on GaAs, pp. 101-109.

${ }^{19} \mathrm{~F}$. Stern, IEEE J. Quanturn Electron. 9, 290 (1973).

${ }^{20}$ D. Botez, Appl. Phys. Lett. 35, 57 (1979).

${ }^{21}$ R. P. Salathe, Appl. Phys. 20, 1 (1979).

${ }^{22} \mathrm{H}$. Kressel and J. K. Butler, Semiconductor Lasers and Heterojunction $L E D$ 's (Academic, New York, 1977), pp. 472.

${ }^{23}$ F. P. Kapron, N. F. Borelli, and D. B. Keck, IEEE J. Quantum Electron. 8. 222 (1972).

${ }^{24} \mathrm{M}$. D. Rourke, and H. W. Yen (private communication).

${ }^{25}$ I. Ladany, H. J. Wakstein, R. S. Crandall, and D. R. Patterson, RCA Research Report, PRRL-78-CR-43 March 1979, p. 22.

${ }^{26}$ R. Lang and K. Kobayashi, IEEE J. Quantum Electron. 12, 194 (1976).

${ }^{27}$ K. Y. Lau, L. Figueroa, and A. Yariv (unpublished).

${ }^{2 B}$ J. A. Copeland, Electron. Lett. 14, 908 (1979).

${ }^{29}$ N. G. Basov, IEEE J. Quantum Electron 5, 855 (1968).

${ }^{30}$ T. P. Lee and H. R. Roldan, IEEE J. Quantum Electron. 6, 339 (1970).

${ }^{3 !}$ R. W. Dixon and W. B. Joyce, IEEE J. Quantum Electron. 15, 470 (1979).

${ }^{32}$ J. P. VanDer Ziel, J. L. Merz, and T. L. Paoli, J. Appl. Phys. 7, 4620 (1979).

${ }^{33}$ B. W. Hakki, J. Appl. Phys. 50, 5630 (1979).

${ }^{34} \mathrm{~L}$. Figueroa, C. Slayman, and H. W. Yen, "Optical-Microwave Interactions in Semiconductor Devices," Hughes Research Laboratories Report under Contract N00173-78-C-0192, April, 1979.

${ }^{35}$ R. K. Jain and J. P. Heritage, Appl. Phys. Lett. 32, 41 (1978). 